\title{
Validation of an Engineered Cell Model for In Vitro and In Vivo HIF-1a Evaluation by Different Imaging Modalities
}

\author{
A. Lo Dico, ${ }^{1,2,3}$ S. Valtorta, ${ }^{4,5,6}$ C. Martelli, ${ }^{2,7}$ S. Belloli, ${ }^{4,5,6}$ U. Gianelli, ${ }^{1,8}$ D. Tosi, ${ }^{7}$ \\ S. Bosari, ${ }^{1,2,8}$ A. Degrassi, ${ }^{9}$ M. Russo, ${ }^{9}$ I. Raccagni, ${ }^{4,5,6,10}$ G. Lucignani, ${ }^{2,7,11}$ \\ R. M. Moresco, ${ }^{4,5,6}$ L. Ottobrini ${ }^{1,2,6}$ \\ ${ }^{1}$ Department of Pathophysiology and Transplantation, University of Milan, Milan, Italy \\ ${ }^{2}$ Centre of Molecular and Cellular Imaging-IMAGO, Milan, Italy \\ ${ }^{3}$ Doctorate School of Molecular Medicine, University of Milan, Milan, Italy \\ ${ }^{4}$ Tecnomed Foundation and Department of Health Sciences, University of Milan-Bicocca, Milan, Italy \\ ${ }^{5}$ Department of Nuclear Medicine, San Raffaele Scientific Institute, Milan, Italy \\ ${ }^{6}$ Institute for Molecular Bioimaging and Physiology (IBFM), National Research Council (CNR), Milan, Italy \\ ${ }^{7}$ Department of Health Sciences, University of Milan, Milan, Italy \\ ${ }^{8}$ Pathology Unit, IRCCS Ca Granda-Ospedale Maggiore Policlinico Foundation, Milan, Italy \\ ${ }^{9}$ Nerviano Medical Sciences, Nerviano, MI, Italy \\ ${ }^{10}$ Doctorate in Biomedical Technologies, University of Milan-Bicocca, Milan, Italy \\ ${ }^{11}$ Department of Diagnostic Services, Unit of Nuclear Medicine, San Paolo Hospital, Milan, Italy
}

\begin{abstract}
Purpose: The aim of this study was to characterize a cell-based model for the molecular study of hypoxiainducible factor (HIF)-1 $\alpha$ activity, in the context of hypoxia, by means of different imaging techniques.

Procedures: Engineered U251-HRE glioma cells were used to analyze the molecular mechanisms underlying HIF-1 $\alpha$ activity in vitro in relation to luciferase expression. The same cells were orthotopically implanted in mice to evaluate tumor progression and hypoxia induction by bioluminescence imaging, fluorescence imaging, positron emission tomography (PET), and magnetic resonance imaging (MRI). Results: In vitro analyses highlighted the relationship between HIF-1 $\alpha$ and luciferase activity in hypoxic conditions and after pharmacological treatments in U251-HRE cells. Through in vivo studies, it was possible to assess hypoxia establishment in relation to tumor growth by optical imaging, PET and MRI. Conclusions: The findings of this study indicate that the U251-HRE orthotopic murine model can be used to reliably evaluate processes modulating HIF-1 $\alpha$ activity, using both molecular and preclinical non-invasive imaging techniques.
\end{abstract}

Key words: Glioma, Hypoxia, Non-invasive molecular imaging, Reporter gene, Optical imaging, PET, MRI, Tumor model

R. M. Moresco and L. Ottobrini are joint senior authors

Electronic supplementary material The online version of this article (doi:10.1007/s11307-013-0669-0) contains supplementary material, which is available to authorized users.

Correspondence to: R. Moresco; e-mail: moresco.rosamaria@hsr.it

\section{Introduction}

$\mathrm{T}$ umor hypoxia and the consequent stabilization of hypoxia-inducible factors (HIFs) play a critical role in cancer progression [1]. The best-characterized hypoxia- 
regulated molecule is HIF-1, which is composed of two subunits, a constitutive subunit called HIF-1 $\beta$ (or ARNT) and the catalytic and oxygen-dependent subunit, HIF-1 $\alpha$. In normal conditions, HIF-1 $\alpha$ (in the presence of cofactors such as oxygen and iron) is hydroxylated by three prolyl hydroxylases (PHD1,2,3). This allows it to bind to the von Hippel-Lindau tumor suppressor protein (pVHL), which targets HIF-1 $\alpha$ for ubiquitination (through the pVHL/E3 ligase complex) and subsequent degradation.

Conversely, in conditions in which either oxygen or iron levels are low, for example in hypoxia, this regulatory mechanism is inhibited because PHDs cannot hydroxylate HIF-1 $\alpha$ [2-5]; as a result, HIF- $1 \alpha$ is able to escape ubiquitination and translocate into the nucleus, where it rapidly accumulates [6]. Within the nucleus, HIF-1 $\alpha$, by binding to the specific hypoxia-responsive elements (HREs) in a complex with HIF-1 $\beta$, induces the expression of responsive target genes such as proangiogenic factors that, promoting neoangiogenesis and vasculature reorganization, in turn, act on proliferation, expansion, and metastasis [7-9].

Brain tumors, in particular the highly aggressive glioblastoma multiforme (GBM), are characterized by very rapid growth and the presence of extensive necrotic/hypoxic areas within the tumor mass; these features correlate with enhanced resistance to therapy, increased invasiveness, and a poor prognosis for the patient. The U251 GBM cell line and relative intracranial mouse model are known to mimic the salient features of human GBM, also at histopathological level [10-12]. Moreover, at genetic level, the U251 xenograft model has been shown to display similarities with human GBM [13, 14], such as the presence of non-functional mutations in p53 and in the phosphatase and tensin homolog (PTEN, found in $\sim 70 \%$ of advanced human GBM). Since PTEN is a negative regulator of the phosphatidylinositol 3kinase (PI3K)/Akt pathway, its mutation in U251 cells results in hyperactivation of the PI3K/Akt pathway that may, in these cells, contribute to enhancing the stability, translocation, dimerization, and transcriptional activity of HIF- $1 \alpha$, even though this event is context and cell type dependent. In fact, analyses of human glioma tissues have suggested a strong correlation between the expression of HIF-1 and malignancy, as described by the World Health Organization tumor grading system [15-17].

Having established the key role of HIF-1 in GBM, it could be useful to clarify the molecular processes regulating its activity, as these could be promising targets for new treatments.

Molecular imaging techniques allow early detection of tumors and the characterization and quantification of biological processes in vitro and in vivo. In animal models, non-invasive in vivo imaging techniques are of particular interest, especially for use in longitudinal multiparametric studies. Among the different available imaging techniques, in vivo optical imaging, herein bioluminescence imaging (BLI) and fluorescence imaging (FLI), is a sensitive and easily accessible means of evaluating tumor progression and hypoxia induction [18]. The possibility of comparing preclinical optical imaging data with data obtained using clinically available techniques such as positron emission tomography (PET) and magnetic resonance imaging (MRI) could open the way for understanding the molecular events occurring at the tumor site and their importance for patient stratification and tumor therapy.

From this perspective, in vivo studies of hypoxia-related molecular processes in the U251 cell line could make a major contribution to the setting up of a GBM model that, allowing stratification of the population on the basis of a significant molecular process, could be used for the in vivo evaluation of new treatments [19].

This study was conducted to validate a U251 engineered cell line (U251-HRE) expressing the luciferase reporter gene under the control of a hypoxia-inducible promoter, as a tool for the noninvasive in vivo study of HIF-1 $\alpha$ activity by BLI $[20,21]$. The aim, in particular, was to demonstrate, through this model, that modulation of HIF-1 $\alpha$ activity by hypoxia or specific treatments can be visualized by non-invasive techniques, using luciferase activity as a hypoxia biosensor. The use of BLI was expected to shed light on the relationship between tumor growth, tumor hypoxia, HIF-1 stabilization, and HIF-1 transcriptional activity in a U251 glioma model. As control, U251-pGL3, constitutively expressing the same reporter (firefly luciferase), was used (Fig. 1a) [22]. The in vivo use of different imaging techniques (BLI, FLI) allowed longitudinal and non-invasive monitoring of the above processes in an orthotopic glioma model. Finally, in order to accelerate the translation of preclinical results into clinical practice, data were cross-validated using imaging techniques adequate for clinical practice and research, namely, PET and MR imaging.

In conclusion, this preclinical model and the powerful imaging strategy proposed herein could be useful for the validation of this cell model and of luciferase activity as an HIF- $1 \alpha$ activity biosensor with a view to future assessments of HIF-1-related antineoplastic treatments.

\section{Materials and Methods}

\section{Cell Lines and Reagents}

Engineered U251-pGL3 and U251-HRE human glioma cells were kindly provided by Dr. Giovanni Melillo, National Cancer Institute, Frederick (MD). U251-pGL3 cells express the luciferase reporter gene under the control of SV40 constitutive promoter and enhancer sequences, whereas U251-HRE cells express the luciferase reporter gene under the control of three copies of an HRE sequence, (pGL2Tk-HRE). We routinely maintained both cell lines in RPMI supplemented with $10 \%$ heat-inactivated fetal bovine serum, penicillin and streptomycin $(50 \mathrm{IU} / \mathrm{ml}), 2 \mathrm{mM}$ glutamine (all Euroclone, Milan, Italy), and $2 \mu \mathrm{l} / \mathrm{ml} \mathrm{G} 418$ (Sigma-Aldrich, St. Louis, MO, USA) in a humidified atmosphere of $5 \%$ of $\mathrm{CO}_{2}$ at $37{ }^{\circ} \mathrm{C}$. 


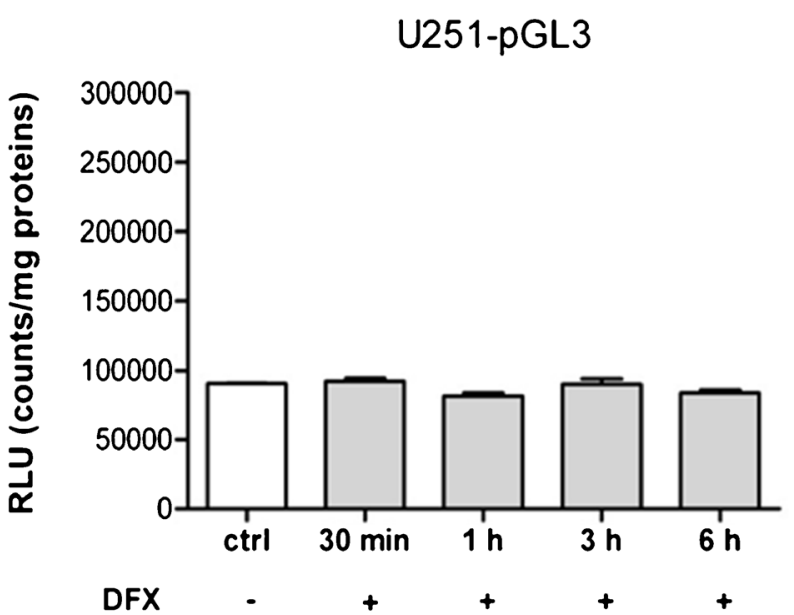

b

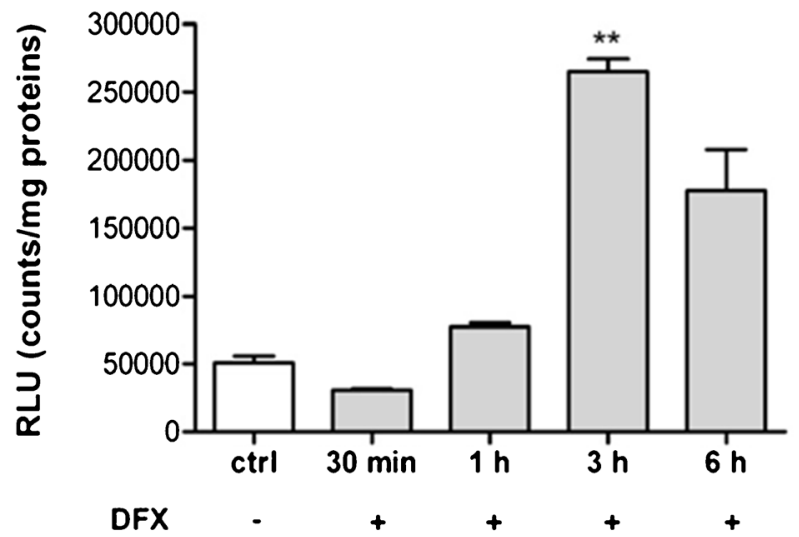

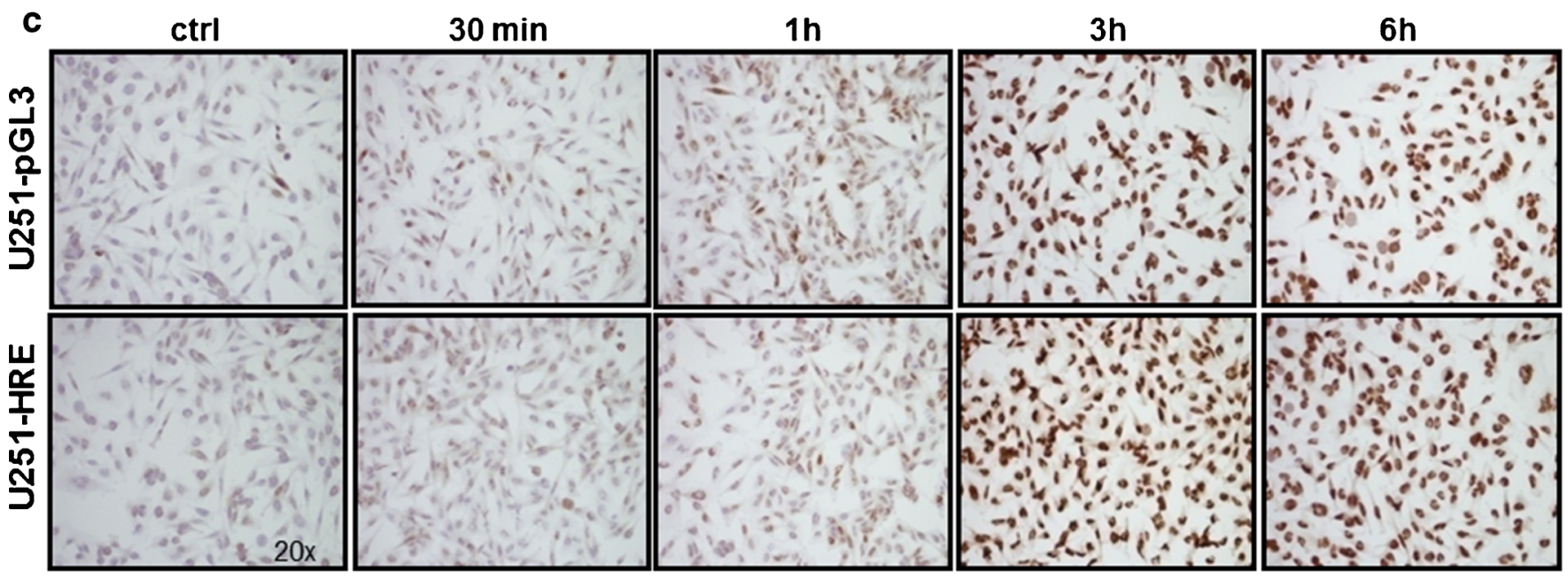

d

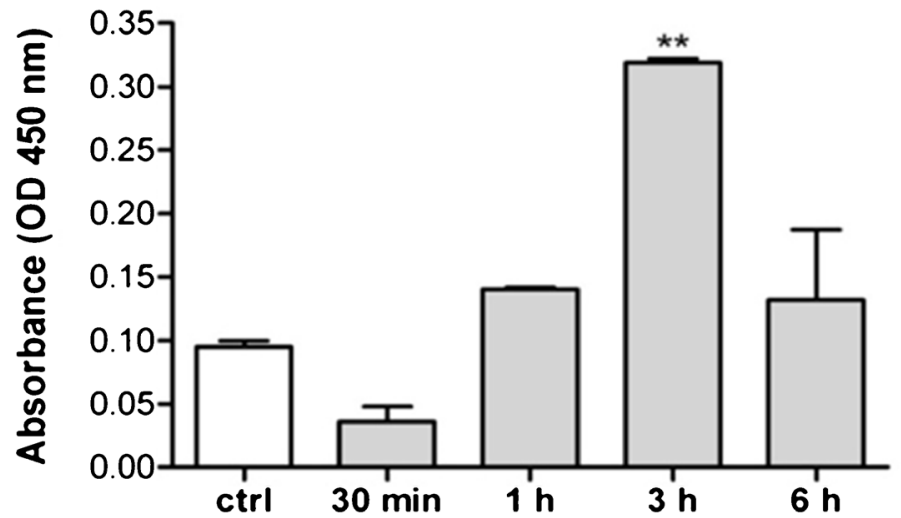

DFX

Fig. 1. In vitro evaluation of luciferase activity in relation to DFX treatment. All experiments were performed at $30 \mathrm{~min}, 1 \mathrm{~h}, 3 \mathrm{~h}$, and $6 \mathrm{~h}$ after treatment. a Luciferase activity after DFX $(100 \mu \mathrm{M})$ treatment in U251-pGL3 cells. The control samples were comprised of untreated cells. Data are expressed as RLU (luciferase counts normalized to amount of proteins quantified by Bradford assay). Data are the mean \pm SE of three independent experiments. A $p$ value $\leq 0.05$ was considered significant. $\mathbf{b}$ Luciferase activity analyzed in U251-HRE cells after DFX $(100 \mu \mathrm{M})$ treatment. DFX vs ctrl ${ }^{* *} p<0.01$. c ICC for HIF-1a both in U251-pGL3 and in U251-HRE cells, performed after DFX $(100 \mu \mathrm{M})$ treatment. The cells were analyzed by optical microscopy at $\times 20$ original magnification (Leica DMD108 microscope). d Nuclear quantification of HIF-1a using an ELISA-based kit in U251HRE cells after DFX $(100 \mu \mathrm{M})$ treatment. Data are expressed as absorbance at $450 \mathrm{~nm}$. Data are the mean \pm SE of three independent experiments. DFX vs ctrl ${ }^{* *} p<0.01$. 


\section{Hypoxia Mimetic Treatment}

To induce hypoxia, cells were treated with $100 \mu \mathrm{M}$ of the hypoxia mimetic agent deferoxamine (DFX, Sigma-Aldrich, St. Louis, MO, USA) at different time points ( $30 \mathrm{~min}, 1 \mathrm{~h}, 3 \mathrm{~h}$, and $6 \mathrm{~h}$ ). DFX was resuspended in distilled sterile water. Treatments with LY294002 $50 \mathrm{mM}$ and FM19G11 $1 \mu \mathrm{M}$ (both Sigma-Aldrich) were carried out to inhibit PI3K and to increase pVHL effects. Both drugs were resuspended in dimethyl sulfoxide (DMSO) according to the manufacturer's instructions (Sigma-Aldrich).

\section{Immunocytochemistry Assay}

Three hundred thousand cells per well in $300 \mu$ l of complete medium were seeded in a chamber slide (Lab-Tek chamber; VWR International PBI Srl, Milan, Italy). The cells were treated with DFX, LY294002, and FM19G11 alone or in combination at different time points (30 min, $1 \mathrm{~h}, 3 \mathrm{~h}$, and $6 \mathrm{~h}$ ) or with DMSO alone as control. At the end of the treatment, the medium was removed and the cells were fixed with cytofix (J.T. Baker, Phillipsburg, NJ, USA). The cells were treated according to the manufacturer's recommended protocol with the primary monoclonal anti-HIF- $1 \alpha$ antibody (54, BD Transduction Laboratories, San Diego, CA, USA). Reactions were revealed by a Novolink Max polymer detection system (Leica Biosystems, Germany), according to the manufacturer's instructions. The chromogen diaminobenzidine (DAB) was incubated for $8 \mathrm{~min}$ at room temperature (RT).

\section{Luciferase Assay}

The in vitro luciferase activity profile was assessed in living cells and in cell lysates before and after treatment with $100 \mu \mathrm{M} \mathrm{DFX}$, $50 \mathrm{mM} \mathrm{LY294002,} \mathrm{and} 1 \mu \mathrm{M}$ FM19G11, alone or in combination, at different time points $(30 \mathrm{~min}, 1 \mathrm{~h}, 3 \mathrm{~h}$, and $6 \mathrm{~h}$ ). A solution containing luciferin $(2 \mathrm{mM})$ was added to the living cells growing in a 96-well plate. Luciferase activity was detected after 5 min by cooled charge-coupled device (CCD) camera imaging (IVIS Lumina; PerkinElmer Life Sciences, Inc, Boston, MA, USA). After lysing, cells were analyzed on a luminometer (GloMax-Multi Detection System; Promega, Madison, WI, USA). Data were normalized to milligram of proteins through Bradford assay and expressed as relative luminescence units (RLU $=$ luciferase counts per milligram proteins).

\section{TransAM Kit}

An ELISA-based kit was used to detect and quantify HIF-1 $\alpha$ transcriptional factor activity. The 96-well plate was coated with oligonucleotides containing the HRE sequences. Nuclear extracts were prepared using the Nuclear Extract Kit (Vinci-Biochem, Florence, Italy) and, after quantification, the samples were added to the coated plate and analyzed using the TransAM Kit (VinciBiochem). Data were expressed as HIF-1 $\alpha$ protein in nuclear extract (OD $450 \mathrm{~nm}$ ).

\section{Animal Studies}

Animal experiments were carried out in compliance with the institutional guidelines for the care and use of experimental animals, which have been notified to the Italian Ministry of Health and approved by the ethics committee of the University of Milan. An orthotopic murine model was obtained by stereotaxic injection (coordinates $1.5 \mathrm{~mm}$ lateral to the bregma, $0 \mathrm{~mm}$ behind, and $3.0 \mathrm{~mm}$ ventral to the dura) [23] of $10^{5}$ glioma cells (U251-HRE and U251-pGL3) in $2 \mu \mathrm{l}$ of PBS into 7-8-week-old female nude mice (Harlan Laboratories, Indianapolis, IN, USA) at day 0.

\section{In Vivo Imaging Study Design}

Different groups of animals were monitored by the following:

- BLI/FLI ( $n=20$; CCD camera, IVIS Lumina, and IVIS Spectrum CT; PerkinElmer Life Sciences)

- PET with 2-deoxy-2- $\left[{ }^{18} \mathrm{~F}\right]$ fluoro-D-glucose $\left(\left[{ }^{18} \mathrm{~F}\right] \mathrm{FDG}\right)$, $3^{\prime}-$ deoxy- $3^{\prime}-\left[{ }^{18} \mathrm{~F}\right]$ fluorothymidine $\left(\left[{ }^{18} \mathrm{~F}\right] \mathrm{FLT}\right)$, and $\left[{ }^{18} \mathrm{~F}\right]$ fluoroazomycin-arabinofuranoside $\left(\left[{ }^{18} \mathrm{~F}\right] \mathrm{FAZA}\right)$ to study glucose metabolism, cell proliferation, and tumor hypoxia, respectively ( $n=6$; YAP(S)-PET II; I.S.E. s.r.l., Pisa, Italy)

- 7 T MRI ( $n=6$, Pharmascan; Bruker Biospin, Ettlingen, Germany)

Starting from cell implantation, BLI scans were performed weekly until the end of the protocol (30 days), while PET and MRI scans were carried out at intermediate (18-20 days) and late (2830 days) time points (Fig. 3a).

\section{BLI/FLI Study}

Briefly, for the detection of bioluminescence, mice were anesthetized with $4 \%$ chloral hydrate $v / v$ (Sigma-Aldrich) and then injected intraperitoneally (i.p.) with $150 \mathrm{mg} / \mathrm{kg}$ of luciferin (Beetle Luciferin Potassium Salt; Promega, Madison, WI, USA). After $20 \mathrm{~min}$ of biodistribution, the mice were placed in the light-tight chamber and the photon emission was then acquired for $10 \mathrm{~min}$. Images were analyzed and scaled after completion of all acquisitions, using appropriate computer software (Living Image Software; PerkinElmer Life Sciences). The same region of interest (ROI) was applied on all the bioluminescent tumors. Data were expressed as average radiance (photons/second/square centimeter/steradian), which is a calibrated measurement of photon emission. This value was normalized to in vitro basal luciferase average radiance measured in both cell lines.

Animals were also submitted to 3D diffuse luminescence imaging tomography (DLIT), performed weekly using the IVIS Spectrum CT, and to fluorescence imaging tomography (FLIT), performed only at the latest time point (30 days), using the HypoxiSense680 fluorescent probe (PerkinElmer Life Sciences), a carbonic anhydrase IX (CAIX)-targeted fluorescent in vivo imaging agent. Both acquisitions were supplemented with an integrated CT scan. 
Twenty-four hours before the FLIT acquisition, the animals were intravenously injected with the HypoxiSense680 probe ( $2 \mathrm{nmol}$ in $100 \mu \mathrm{l}$ of sterile PBS). On the following day, they were injected i.p. with $150 \mathrm{mg} / \mathrm{kg}$ of luciferin and, after biodistribution, submitted to DLIT and FLIT acquisition. Images were analyzed with Living Image ${ }^{\circledR}$ software (PerkinElmer Life Sciences) using volumetric ROI analysis. Data were expressed as average radiance for DLIT reconstruction and as radiant efficiency for FLIT.

\section{MRI and PET Analysis}

MRI was performed on a Bruker Pharmascan system equipped with a 7.0T horizontal magnet. Scans were acquired at intermediate and later time points after intracranial cell injection.

Animals were anesthetized with gas ( $2 \%$ isoflurane), positioned prone on the animal bed and inserted into the radiofrequency coil (diameter of $38 \mathrm{~mm}$ ) inside the magnet. Scout transverse and sagittal images were acquired for correct positioning of the ROI. Different MR sequences were used in order to optimize intracranial tumor visualization and contrast.

Anatomical 2D multi-slice turbo spin echo sequences (RARE; field of view (FOV), $2.5 \times 2.5 \mathrm{~cm}$; matrix, $256 \times 256$; $0.8-\mathrm{mm}$ slice thickness; TR/TE, 4,200/14 ms; TEeff, $42 \mathrm{~ms}$; RARE factor, 8; four averages; acquisition time, $6 \mathrm{~min}$ and $43 \mathrm{~s}$ ) were run in both transverse and coronal orientations.

Diffusion MRI studies, able to detect necrotic regions in tumors, were performed in the axial orientation with a diffusion-weighted imaging-echo planar imaging (DWI-EPI) sequence [FOV, $2.5 \times$ $2.5 \mathrm{~cm}$; matrix $128 \times 128$; TR/TE, $3,000 / 36 \mathrm{~ms}$; two averages; $5 \mathrm{~A} 0$, three directions, three $b$ values per direction $(b=400,1,100$, $1,800 \mathrm{~s} / \mathrm{mm} 2 ; \delta=7 \mathrm{~ms}, \Delta=16 \mathrm{~ms}$; acquisition time, $2 \mathrm{~min}$ and $48 \mathrm{~s})]$.

After MRI scanning, mice were also evaluated using a YAP-(S)PET II small-animal scanner with $\left[{ }^{18} \mathrm{~F}\right] \mathrm{FDG},\left[{ }^{18} \mathrm{~F}\right] \mathrm{FLT}$, and $\left[{ }^{18} \mathrm{~F}\right] \mathrm{FAZA}$ on consecutive days at intermediate and later time points. $\left[{ }^{18} \mathrm{~F}\right] \mathrm{FDG}$ was prepared in our facility for clinical use as indicated in the European Pharmacopeia, VII ed., while $\left[{ }^{18} \mathrm{~F}\right] \mathrm{FAZA}$ and $\left[{ }^{18} \mathrm{~F}\right] \mathrm{FLT}$ were obtained as previously described $[24,25]$, with minor modifications. All the radiopharmaceuticals injected had a radiochemical purity greater than $99 \%$. Animals in fasting condition were injected in a tail vein with $4.41 \pm 0.22 \mathrm{MBq}$ of $\left[{ }^{18} \mathrm{~F}\right] \mathrm{FDG}, 4.28 \pm 0.24 \mathrm{MBq}$ of $\left[{ }^{18} \mathrm{~F}\right] \mathrm{FLT}$, and $5.72 \pm 0.31 \mathrm{MBq}$ of $\left[{ }^{18} \mathrm{~F}\right] \mathrm{FAZA}$. Under light anesthesia with ether, the mice were positioned prone on the tomograph bed with the head centered in the tomograph FOV. The animals were positioned using a special polystyrene support in order to allow co-registration of the images obtained with the three radiotracers. Dynamic acquisitions with $\left[{ }^{18} \mathrm{~F}\right] \mathrm{FDG}$ and $\left[{ }^{18} \mathrm{~F}\right] \mathrm{FLT}$ started $60 \mathrm{~min}$ after injection and lasted $30 \mathrm{~min}$ (six scans of $5 \mathrm{~min}$ each), whereas scans with $\left[{ }^{18} \mathrm{~F}\right] \mathrm{FAZA}$ started $120 \mathrm{~min}$ after injection and lasted $15 \mathrm{~min}$ (three scans of 5 min each). Throughout the scans, the animals were kept under gas anesthesia with $2 \%$ isoflurane.

PET images were acquired in 3D mode and reconstructed using the expectation-maximization algorithm. All the images were calibrated with a dedicated phantom, corrected for the isotope half-life, and then quantified with PMOD 2.7 software (Zurich, Switzerland). The radiotracer uptake of tumoral and background regions measured using ROI analysis was as follows: circular ROIs were drawn on the whole tumor mass using the automatic isocontour tool of the PMOD software. Background regions were defined by manually drawing circular ROIs on three consecutive transaxial slices on a contralateral control region (volume of ROIs, $\left.0.5 \pm 0.1 \mathrm{~mm}^{3}\right)$ for $\left[{ }^{18} \mathrm{~F}\right] \mathrm{FDG}$ and on the cerebellum for $\left[{ }^{18} \mathrm{~F}\right] \mathrm{FAZA}$ and $\left[{ }^{18} \mathrm{~F}\right] \mathrm{FLT}$ (volume of ROIs, $9.7 \pm 2.9 \mathrm{~mm}^{3}$ ). Radioactivity concentration was calculated as maximum standardized uptake value $\left(\mathrm{SUV}_{\max }\right)$ correcting for injected dose and animal weight, according to the formula $\mathrm{SUV}=$ (region of radioactivity/injected radioactivity) $\times$ animal weight. Uptake data were also calculated as tumor-to-background ratio $(T / B)$ dividing the $\mathrm{SUV}_{\max }$ of the tumor by the $\mathrm{SUV}_{\text {mean }}$ of the background region. PET images were shown as SUV images after correcting for injected activity and for body weight of animals.

\section{Immunohistochemistry}

Following the last acquisition (at 30 days), the animals were sacrificed and their brains were collected, fixed in $10 \%$ neutral buffered formalin (Sigma-Aldrich), and paraffin-embedded after dehydration with increasing concentrations of ethanol $(75,85,95$, and $100 \%, 9$ min each) and xylene rinse.

Longitudinal sections (3-4 $\mu \mathrm{m})$ were cut and stained with hematoxylin and eosin (H\&E) for the morphological evaluation. The deparaffinized sections were immunohistochemically assessed using the Genomix i-6000 automated staining system (BioGenex, San Ramon, CA). Heat-induced antigen unmasking was performed using either EDTA $(0.05 \mathrm{M}, \mathrm{pH} 8)$ or citrate $(0.01 \mathrm{M} \mathrm{pH}$ 6) solutions in a thermostatic bath for $35 \mathrm{~min}$. After cooling, slides were washed in buffer and then incubated for $1 \mathrm{~h}$ at RT with the following antibodies: monoclonal anti-HIF-1 $\alpha$ antibody (clone 54, dilution 1:200 in EDTA, BD Transduction Laboratories, San Diego, CA); monoclonal anti-CAIX antibody (ab15086, dilution 1:6,000, $1 \mathrm{~h}$ in citrate $\mathrm{pH}$ 6, Abcam, Cambridge, UK); monoclonal mouse anti-human Ki-67 antigen (clone MIB-1, dilution 1:100 in citrate, Dako, Hamburg, Germany); and anti-firefly luciferase antibody (ab498, Abcam, Cambridge, UK). Reactions were revealed using the Novolink Max polymer detection system (Leica Biosystems), in accordance with the kit manufacturer's instructions, with the polymer for $30 \mathrm{~min}$ and with DAB for $8 \mathrm{~min}$.

\section{Statistical Analysis}

Both in vitro and in vivo experiments were repeated at least twice giving reproducible results. Data are presented as mean values \pm standard deviation. Statistical analysis was performed using Prism 4 (GraphPad Software Inc., San Diego, CA, USA); one-way ANOVA (nonparametric) was performed, followed by Dunnett's multiple comparison test.

\section{Results}

\section{In Vitro Characterization of Luciferase Activity After HIF-1 $\alpha$ Modulation}

The luciferase activity profile was assessed in vitro in cell lysates, before and after treatment with the hypoxia mimetic DFX, to characterize the influence of HIF-1 $\alpha$ stabilization on 
reporter gene expression in the two cell lines. In accordance with Rapisarda et al. [20, 22], we confirmed that, after hypoxia mimetic treatment, luciferase activity in U251pGL3 cells was independent of HIF- $1 \alpha$ modulation, whereas only U251-HRE cells expressed the luciferase reporter gene in relation to HIF-1 $\alpha$ nuclear accumulation. Luciferase activity was seen to increase after DFX treatment in U251HRE cells, reaching the highest signal after $3 \mathrm{~h}$ of treatment and decreasing thereafter. Conversely, luciferase activity did not change in U251-pGL3 cells after treatment with DFX (Fig. 1a, b). Proliferation assay (MTT) confirmed the absence of detectable differences between the two cell lines in terms of DFX toxicity and influence on cell proliferation (data not shown). The effects of DFX treatment were also analyzed by immunocytochemistry assay (ICC) and through quantification of HIF-1 $\alpha$ in nuclear extracts. The ICC of HIF-1 $\alpha$ showed no differences in translocation kinetics between the two cell lines, confirming a peak of nuclear translocation at $3 \mathrm{~h}$ after DFX treatment (Fig. 1c). This result was also confirmed by a quantitative assay of HIF- $1 \alpha$ in nuclear extracts, which showed it to translocate into the nucleus after DFX treatment with the same kinetics already observed in ICC (Fig. 1d). The effects of PI3K inhibitor (LY294002) and HIF-1 $\alpha$ inhibitor (FM19G11), in living and lysated cells, were also investigated on the basis of the pathway reconstructed in Fig. 2a [26].

Figure $2 b, c$ show luciferase activity, as a result of the transcriptional effect mediated by HIF-1 $\alpha$, after treatments with LY294002 and FM19G11, respectively. LY294002 treatment had no relevant influence at the early time points (30 min and $1 \mathrm{~h}$ ), whereas at $3 \mathrm{~h}$, in the presence of DFX, LY294002 treatment was found to counteract hypoxia mimetic treatment-mediated luciferase induction. At $6 \mathrm{~h}$, the effect of LY294002 decreased, since HIF-1 $\alpha$ availability was reduced (probably due to negative transcription-dependent feedback). On the other hand, FM19G11-mediated inhibition completely abolished DFX-mediated luciferase induction, even in the presence of DFX. Luciferase activity modulation was also visualized and confirmed by CCD camera imaging in living cells, demonstrating that this instrument can be used to assess bioluminescence variation due to different treatments (Fig. 2d).

\section{In Vivo Optical Imaging of Luciferase Activity in Relation to Intratumoral Hypoxia}

To clarify the meaning of luciferase expression in orthotopic models obtained using U251-pGL3 and U251-HRE cells, in vivo longitudinal studies showing the bioluminescent signal over time were performed: animals were imaged immediately after cell injection and then weekly to monitor hypoxia establishment and development (Fig. 3a, b). Only in orthotopic U251-pGL3 models was luciferase activity found to be detectable immediately after injection and to progressively increase over time in relation to tumor growth, showing a linear trend for the entire period of observation. In U251-HRE orthotopic models, the luciferase activity linearity was not maintained through to the late time point. Indeed, starting from day 18 , luciferase activity showed a sudden increase, changing the slope of the expected linear progression (Fig. 3c).

This difference in the pattern of luciferase activity increase between the tumor models was shown by the in vivo average radiance, normalized to cellular basal luciferase activity. Indeed, even though MRI showed slower growth kinetics in U251-HRE than in U251-pGL3-derived tumors (data not shown), in the U251-HRE model, the trend line reporting luciferase activity over time showed a steep increase with respect to the linear signal observed in U251-pGL3 (Fig. 3c), probably due to hypoxia establishment within the implanted tumor. This underlines once more the dependence of luciferase expression on hypoxia. In view of these findings, subsequent studies concentrated on U251-HRE cells, with U251-pGL3 cells having been used as controls for the initial evaluations.

Luciferase activity in mouse models was also analyzed in relation to the accumulation of a specific hypoxia probe. Mice were injected with HypoxiSense680 fluorescent probe and scanned for luciferase expression using 3D FLIT and DLIT. Volumetric ROI analysis of the DLIT images allowed tumor location and dimension to be determined (mean volume $=$ $11.97 \pm 3.1 \mathrm{~mm}^{3}$, depth $=2.3 \mathrm{~mm} \pm 0.1$ ). The FLIT acquisition showed an extensive hypoxic area co-localizing with the bioluminescent signal (mean volume $=9.34 \pm 2.8 \mathrm{~mm}^{3}$ ) (Fig. 4).

\section{Morphological and Immunohistochemical Analyses}

To validate GBM features and imaging data, morphological and immunohistochemical (IHC) analyses were performed on explanted brains. Ex vivo $\mathrm{H} \& \mathrm{E}$ staining showed distinctive features of high-grade glioma (i.e., GBM), namely, markedly hypercellular proliferation of spindle and/or fusiform cells, with atypical and pleomorphic nuclei, high mitotic activity, prominent microvascular proliferation, and necrosis. Moreover, the U251-HRE cells showed an infiltrative pattern of invasion into normal brain parenchyma, illustrating the

Fig. 2. Luciferase activity after treatments modulating the HIF1a pathway. a Schematic representation of the PI3K/Akt pathway and treatment targets. b U251-HRE cells underwent both single and double treatments with DFX $(100 \mu \mathrm{M})$ and/or LY294002 (50 mM), for analysis at the time points previously mentioned. The dashed line indicates luciferase activity in untreated cells (ctr). Data are expressed as RLU. Data are the mean \pm SE of three independent experiments. ${ }^{*} p<0.05$, ${ }^{* *} p<$ $0.01,{ }^{* \star *} p<0.001$. c U251-HRE cells underwent both single and

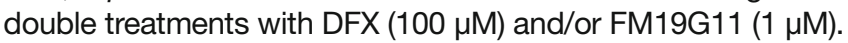
The cells were analyzed as previously described. Data are the mean \pm SE of three independent experiments. ${ }^{*} p<0.05$, ${ }^{* *} p<$ $0.01,{ }^{* *} p<0.001$. d Luciferase activity was analyzed in living U251-HRE cells by CCD camera after treatments with DFX

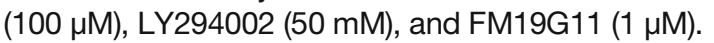


a

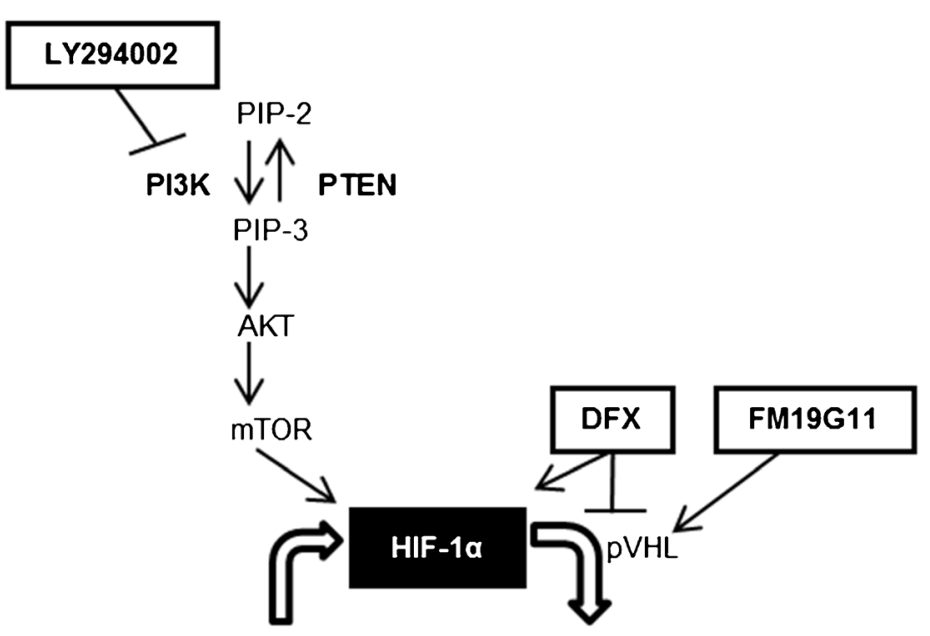

mRNA transcription

Proteosomic degradation

b

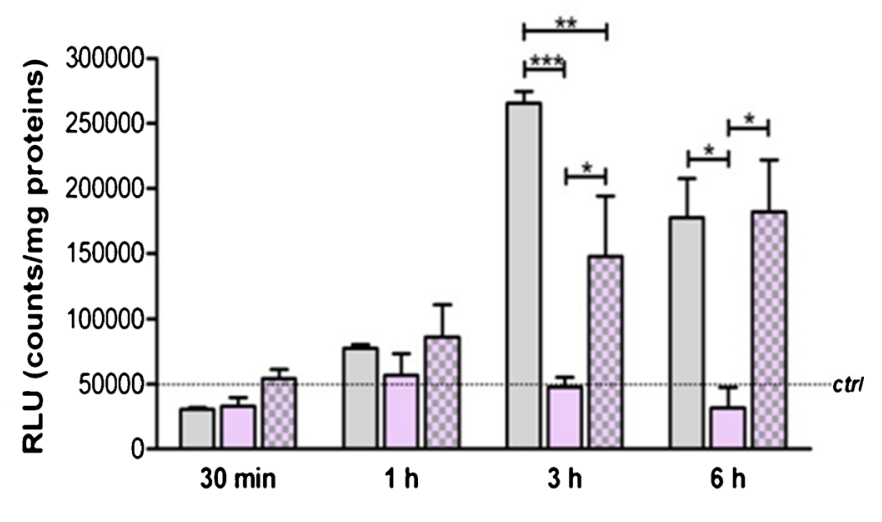

DFX
LY29400
C

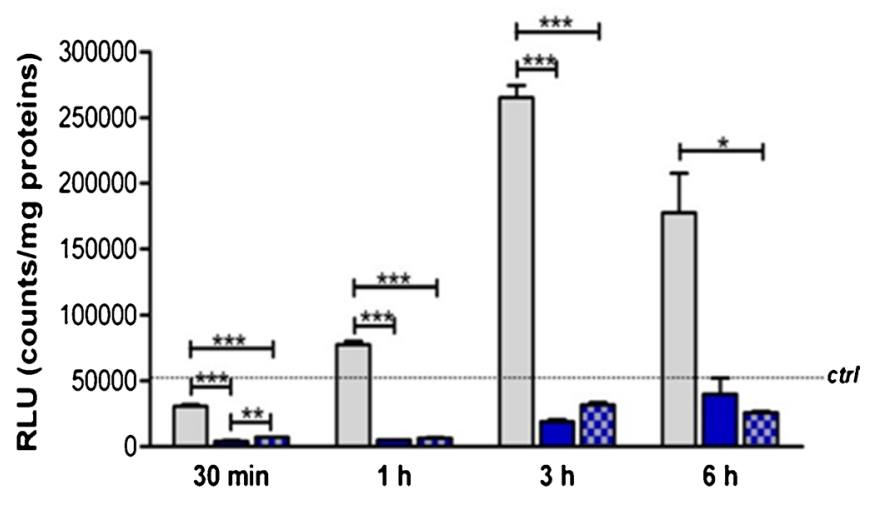

d
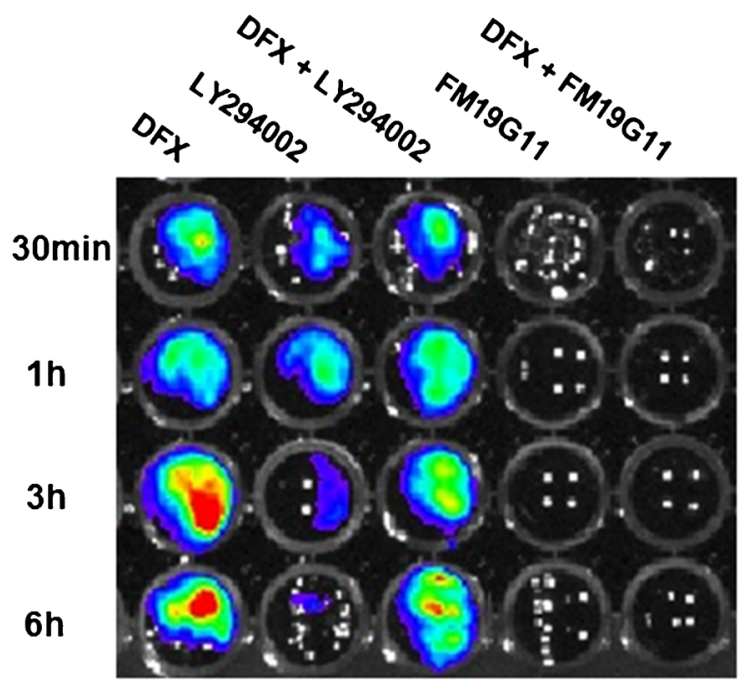

Min Max 


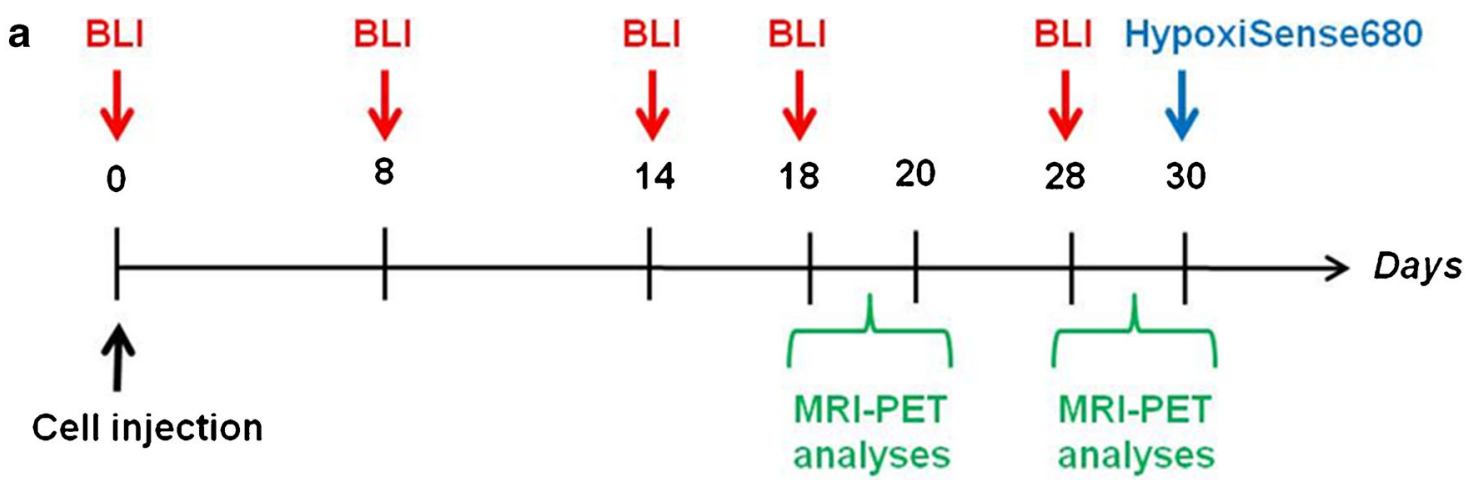

b

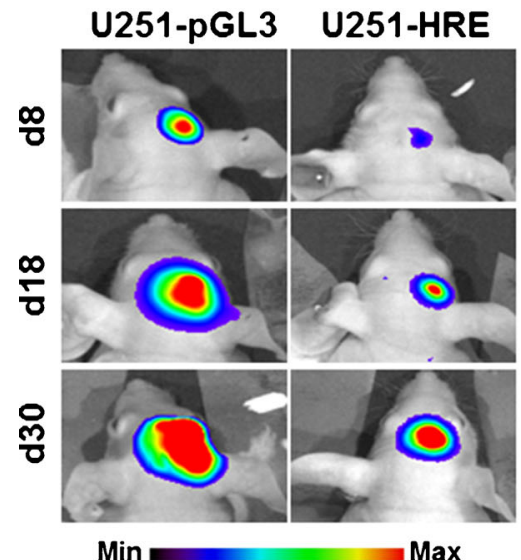

C

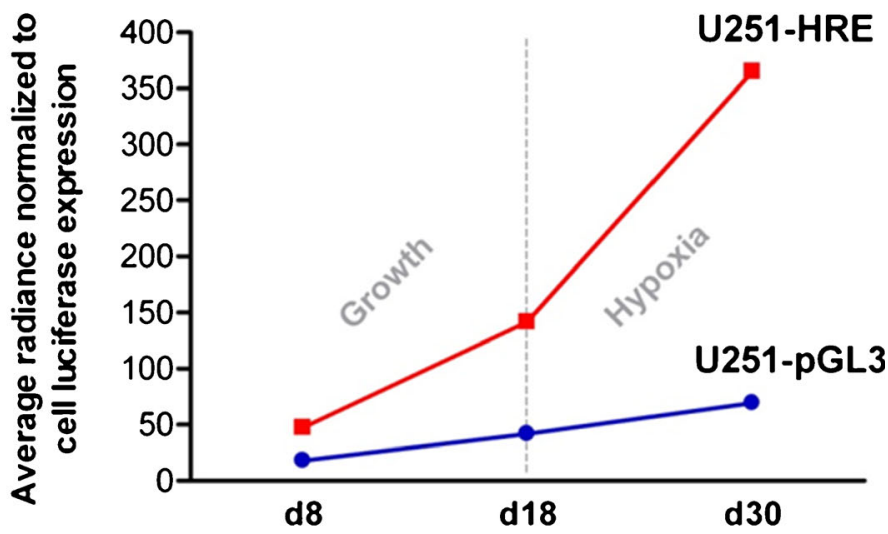

Fig. 3. In vivo detection of luciferase activity in orthotopic glioma models. a Time line of the study design. b 2D BLI images in U251-pGL3 and U251-HRE tumor models analyzed at 8, 18, and 30 days after cell injection. Images are presented with the same scale bar. c Graphical representation of luciferase activity over time in the two orthotopic models. ROls were drawn on 2D images and the resulting luciferase activities, expressed as average radiance (photons/second/square centimeter/steradian), were normalized to basal luciferase activity for each cell line.

aggressiveness of tumor cells (Fig. 5a). IHC showed a strong positivity for hypoxia markers: nuclear HIF-1 $\alpha$ and membrane CAIX were observed, in keeping with the presence of hypoxia in the tumors $[11,20]$. Ki67 staining was strongly positive, reflecting the high proliferation index of tumor lesions. Finally, IHC for luciferase expression supported the results of the in vivo bioluminescence analysis. Indeed, positive cytoplasmic staining of luciferase was found to colocalize with HIF-1 $\alpha$ nuclear positivity in adjacent sections (Fig. 5b).

\section{In Vivo Study of Tumor Features by MRI and PET}

MRI was performed to document, in detail, the localization and growth of tumor masses. On anatomical RARE images, the U251-HRE tumor was delineated from normal brain tissue as an isointense or slightly hyperintense, poorly demarcated, and irregularly rounded area. Furthermore, MRI diffusion experiments were performed to analyze the relative diffusion coefficient in necrotic and nonnecrotic tumor areas. Figure 6a shows some examples of color-coded diffusion maps (DWI-EPI) (1-3) and the corresponding anatomical RARE images (2-4). At 20 days, very few areas of internal necrosis were found and diffusion maps appeared mostly homogeneous (1-2); at 30 days, the biggest tumors started showing internal necrotic regions, characterized by a higher diffusion coefficient (3-4).

In order to better characterize tumor growth and discriminate the hypoxia signal in relation to glucose metabolism and cell proliferation, the role of $\left[{ }^{18} \mathrm{~F}\right] \mathrm{FDG}$, $\left[{ }^{18} \mathrm{~F}\right] \mathrm{FAZA}$, and $\left[{ }^{18} \mathrm{~F}\right] \mathrm{FLT}$ in U251-HRE tumors was studied by in vivo PET imaging at 18-20 and 28-30 days after cell implantation (Fig. 6b). $\left[{ }^{18} \mathrm{~F}\right] \mathrm{FDG}$ PET studies revealed no significant increase in uptake either at intermediate $(T / B=$ $1.27 \pm 0.17)$ or at late time points $(1.08 \pm 0.06)$ (ESM 1). On the contrary, U251-HRE animal models showed poorly hypoxic $\left(\left[{ }^{18} \mathrm{~F}\right] \mathrm{FAZA} T / B=1.01 \pm 0.09\right)$ but highly proliferative tumors $\left(\left[{ }^{18} \mathrm{~F}\right] \mathrm{FLT} T / B=1.33 \pm 0.14\right)$ on the intermediate PET studies (18-20 days). At 30 days after cell injection, tumors showed hypoxic areas as indicated by $\left[{ }^{18} \mathrm{~F}\right] \mathrm{FAZA}$ uptake $(T / B$ ratio, $1.38 \pm 0.09)$ and the $\left[{ }^{18} \mathrm{~F}\right] \mathrm{FLT} T / B$ ratio increased significantly to $2.38 \pm 0.15$ (a 1.78 -fold increase, 
DLIT
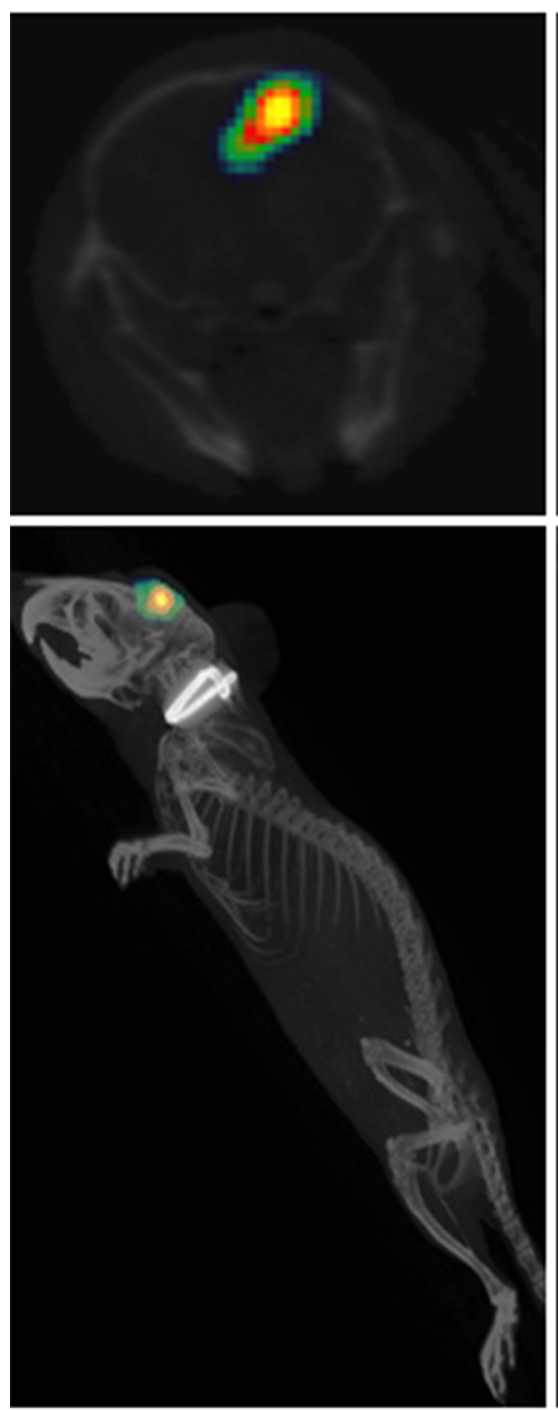

\section{FLIT HypoxiSense680}
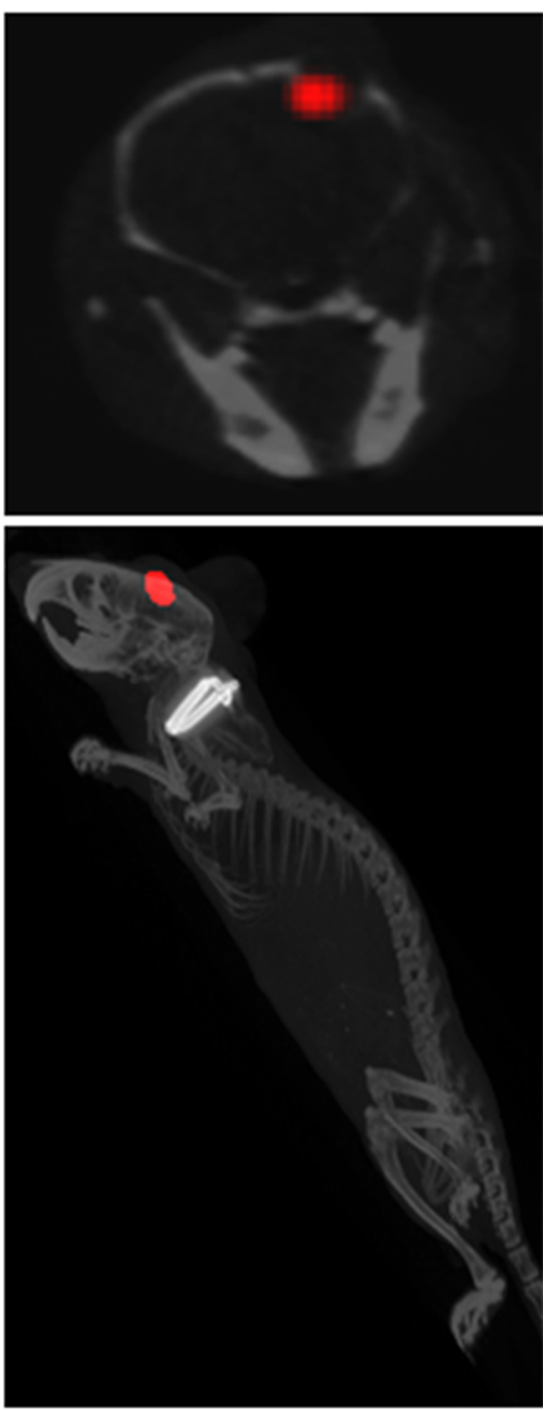

Min

Max Min

Max

Fig. 4. 3D and axial comparison of luciferase activity and HypoxiSense680 fluorescent probe uptake in a representative U251HRE orthotopic mouse model $(n=3)$. Scans were performed at day 30.

$p=0.003$ ). These findings were corroborated by IHC staining as already reported.

\section{Discussion}

Hypoxia is considered to play a crucial role in tumor development and progression and to be a main factor in GBM, promoting angiogenesis, tumor invasiveness, loss of apoptotic potential, genomic instability, and chemotherapy and radiation resistance [27]. For these reasons, hypoxia is considered a promising target for the treatment of many different human cancers usually characterized by the presence of extensive hypoxic and necrotic areas, GBM in particular. In this context, hypoxia and especially the oxygenation-dependent transcription factor, HIF-1, could be valid targets for the development of new cancer therapies. Molecular imaging techniques can be used to non-invasively study hypoxia-related processes in preclinical models, allowing longitudinal studies in the same animal. In this study, luciferase activity was used as a hypoxia biomarker. First, the dependence of reporter gene expression on HIF-1 $\alpha$ activity was demonstrated in vitro and in vivo by BLI and FLI and found to characterize the U251-HRE cell line in relation to the control U251-pGL3 cell line both for tumor growth and hypoxia establishment.

Subsequently, in vivo studies were performed using MRI and PET in order to validate this model using preclinical 


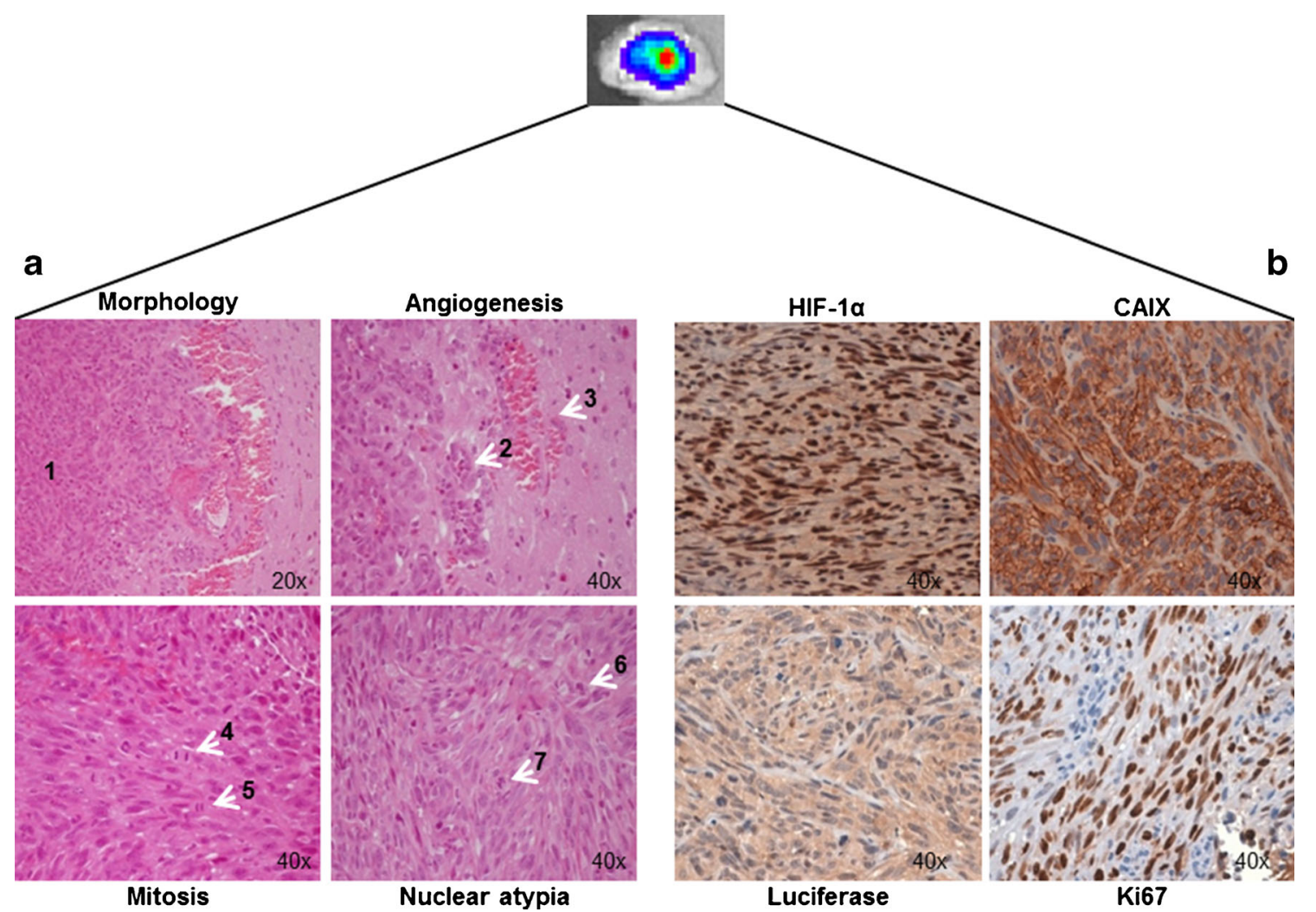

Fig. 5. H\&E staining and IHC validation on ex vivo brain sections. a Ex vivo H\&E staining shows hypercellular neoplastic tissue (1), infiltrating the brain parenchyma. Tumors are characterized by "glomeruloid capillaries" (2) and prominent microvascular proliferation (3); brisk mitotic activity (4-5); nuclear atypia (6), and abnormal mitoses (7). b IHC staining for hypoxia markers (HIF-1a and CAIX), for proliferation marker (Ki67), and for luciferase expression.

imaging procedures potentially transferable to the clinical setting.

These studies were performed both after HIF-1 $\alpha$ induction and after pharmacological modulation using two engineered cell lines (chosen as the simplest model for studying HIF-1 $\alpha$ activity in GBM): U251-HRE cells, expressing the luciferase reporter gene under the control of the HRE sequences, and U251-pGL3 cells, constitutively expressing the luciferase reporter gene as control.

Rapisarda et al. [20-22] have already demonstrated that U251-HRE cells show low but detectable levels of luciferase activity in normoxic condition and that these levels increase under hypoxic conditions. On the contrary, U251-pGL3 cells express the same level of luciferase both in normoxic and hypoxic conditions.

To establish whether changes in HIF-1 $\alpha$ accumulation might induce luciferase activity modulation, both cell lines were first treated with DFX to mimic a hypoxic microenvironment [28]. Only in U251-HRE cells was DFX treatment found to produce a modulation of luciferase expression, corresponding to HIF-1 $\alpha$ stabilization. In particular, a peak of activity was detectable $3 \mathrm{~h}$ after DFX treatment, followed by a decrease at $6 \mathrm{~h}$, probably due to transcription-dependent feedback regulation [29]. Indeed, Stiehl et al. [30] have already demonstrated that HIF-1 $\alpha$ mRNA can induce a negative feedback mechanism, decreasing HIF-1 $\alpha$ stability, and this phenomenon was also observed when cells were chronically exposed to hypoxic conditions. Mottet et al. [31] suggested, on the basis of in vitro studies, that in the presence of prolonged hypoxia the PI3K/Akt pathway activity is reduced, influencing the regulation of glycogen synthase kinase 3 beta (GSK3 $\beta$ ) activity on HIF $1 \alpha$. Indeed, reduction of Akt activity reduces the quantity of the regulatory form of GSK3 $\beta$ phosphorylated on Ser-9 and allows the Tyr-216 phosphorylated form of GSK $3 \beta$, already present in the cell, to reduce the stabilization of HIF- $1 \alpha$ and its transcriptional activity. Although this process can be observed from $6 \mathrm{~h}$ after HIF-1 $\alpha$ induction, it is stronger at a late time point $(16 \mathrm{~h})$. The data herein reported show that DFX-mediated HIF- $1 \alpha$ induction is time dependent $[22,32]$ and suggest that the greatest effect of HIF-1 $\alpha$ is detectable between 3 and $6 \mathrm{~h}$ after treatment, in line with its transcriptional activity. These data were confirmed by ICC of HIF- $1 \alpha$ and by quantification of HIF- $1 \alpha$ in nuclear extracts in both cell lines; however, only in the U251-HRE cells was luciferase activity found to reflect this molecular event.

To establish whether luciferase activity can also reflect a pharmacological modulation of the pathways involved in HIF-1 $\alpha$ activity, in vitro studies with PI3K inhibitors 
a

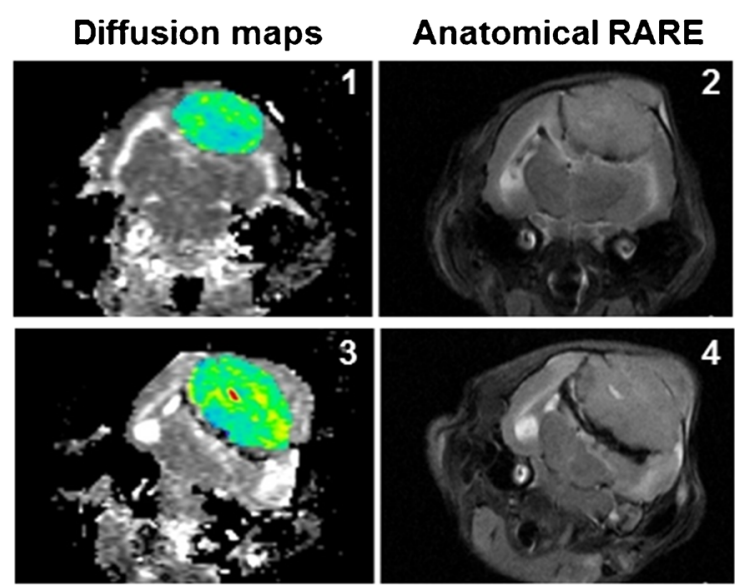

Min $=$ Max

b MRI BLI SUV [18F]FAZA PET SUV [18F]FLT PET
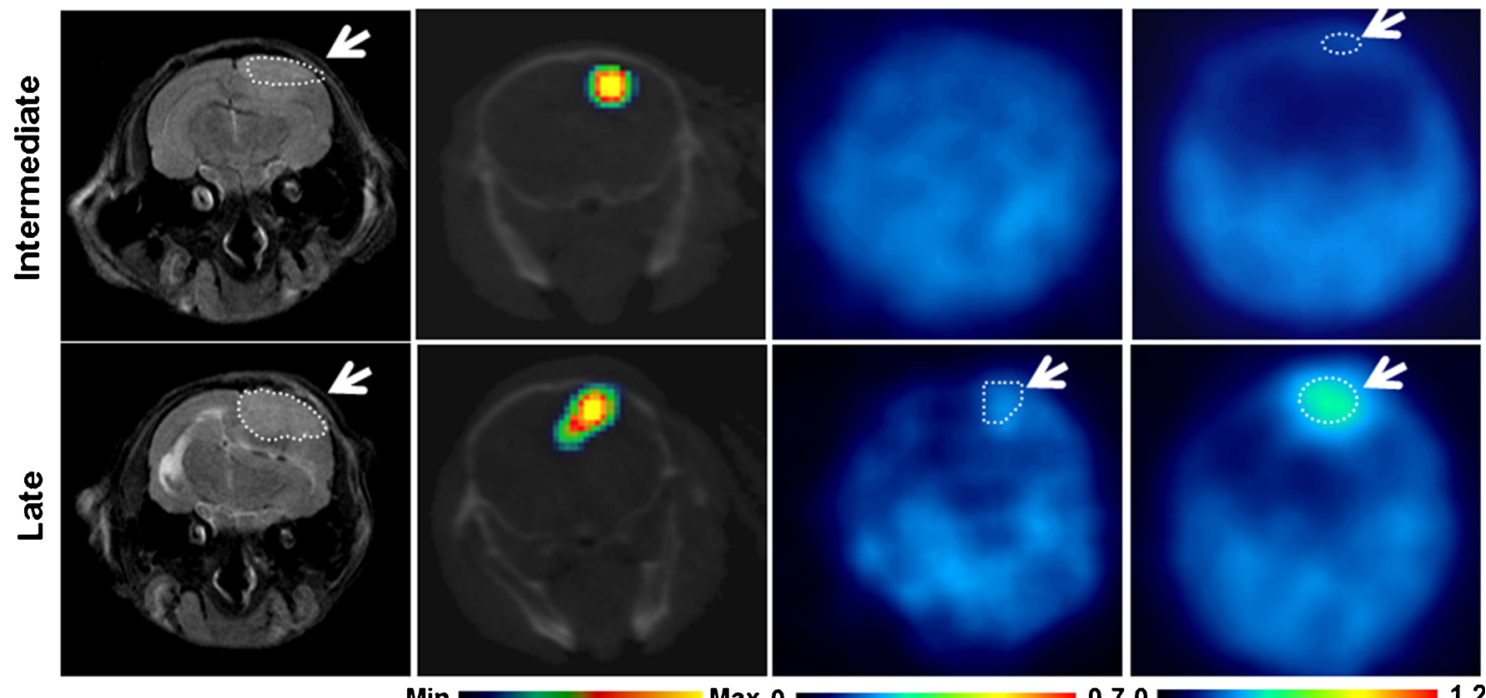

Fig. 6. PET and MRI analyses. a Color-coded MRI diffusion maps $(1,3)$ with corresponding anatomical RARE images $(2,4)$ at 20 days $(1,2)$ and 30 days $(3,4)$ : tumors are encircled by white dotted lines on RARE images. Higher diffusion coefficient values are depicted as yellow or red areas. b Representative axial MRI, BLI, and PET images in U251-HRE animal models performed at intermediate (18-20 days) and late (28-30 days) time points. White arrows indicate tumors. Tumors are delineated by white dotted lines.

(LY294002) or HIF-1 $\alpha$ inhibitors (FM19G11) were performed in U251-HRE cells. There is, in fact, evidence in the literature suggesting that the PI3K/Akt signal transduction pathway has an important role in hypoxia-induced stabilization of HIF-1 $\alpha$ [26, 31, 32] and could therefore be extremely important as a therapeutic target in several tumor types.

Three hours after treatment with LY294002, cells concomitantly treated with DFX, compared with those treated with DFX alone, showed a reduction in luciferase activity. Indeed, only in the presence of DFX, which reduced HIF-1 $\alpha$ degradation, was LY294002 treatment, by inhibiting PI3K, able to reduce Akt-mediated HIF-1 $\alpha$ stabilization and thus to produce a decrease in luciferase activity. On the other hand, at $6 \mathrm{~h}$, both treatment with DFX alone and the double treatment produced comparable luciferase activities. This might be explained by the difference in the timing of the triggering of the negative transcription-dependent pathway in the two different treatments, which depends on the HIF$1 \alpha$ mRNA level, found to be very high at $3 \mathrm{~h}$ after treatment with DFX alone $[26,29]$. On the other hand, direct HIF-1 $\alpha$ inhibition, mediated by FM19G11 treatment, completely abolished DFX-mediated luciferase induction, even in the presence of DFX. All these results were also obtained by CCD camera imaging of luciferase activity in living cells.

Zundel et al. [33] demonstrated that responsiveness of HIF-1 $\alpha$ transcriptional activity to induced hypoxia was increased in the U251 cell line compared with the other 
tumor cells, due to the complex genetic background of this model, characterized by the presence of a mutation in the gene coding for the tumor suppressor PTEN, which plays an important role in the PI3K/Akt pathway, increasing HIF-1 synthesis and activity [7, 22, 34].

Indeed, in standard conditions, PTEN inhibits the PI3K that is necessary to activate the Akt pathway; instead, mutation of the PTEN tumor suppressor gene allows hyperactivation of PI3K/Akt, which leads to HIF-1 $\alpha$ stabilization. Furthermore, PTEN mutation has been found in almost $70 \%$ of advanced GBM in humans [35], where it is correlated with tumor expansion due to apoptotic resistance and to promotion of cell survival, even in a microenvironment not conducive to tumor growth, as also reported by Rong et al. As a consequence, high sensitivity to HIF-1 $\alpha$ and consequent vascular endothelial growth factor induction can be observed, promoting in this condition a positive feedback that drives angiogenic gene expression and tumor progression [36].

Having established, in vitro, that luciferase activity reflected HIF-1 $\alpha$ accumulation, we performed in vivo studies in orthotopic murine models obtained using both U251-HRE and U251-pGL3 cells to examine, by optical imaging, hypoxia induction in relation to tumor growth and luciferase expression. In vivo BLI studies showed that the two cell lines had different luciferase linearity windows. Indeed, while U251-pGL3 cells showed an increase of luciferase activity proportional to cell proliferation, U251-HRE cells showed a bimodal trend of luciferase expression. From day 18 after hypoxia establishment, luciferase basal expression was found to be enhanced due to HIF-1 transcriptional activity. In summary, luciferase expression has a different meaning in the two cell lines: it is related to cell proliferation in U251-pGL3 cells and to HIF-1 $\alpha$ activity in U251-HRE cells, suggesting that this model could be of great interest for the in vivo study of hypoxia-related processes and treatments. The suitability of U251-HRE cells for long-term study of hypoxia has also been confirmed by others [20,21]. In fact, it has been demonstrated that U251-HRE cells are able to maintain stable hypoxia-mediated luciferase activity for long periods of time, making this model suitable for investigation of HIF- $1 \alpha$.

To confirm the meaning of luciferase expression in U251HRE cells, the HypoxiSense680 fluorescent probe was utilized to directly evaluate hypoxic areas within orthotopic tumors. In fact, this probe localizes by binding to CAIX, which is overexpressed in hypoxic tumor areas. The results showed a co-localization of the bioluminescent and fluorescent signals in U251-HRE-injected mice, demonstrating that luciferase activity was indeed induced by the establishment of hypoxia.

The in vivo results were validated by ex vivo IHC staining. In particular, H\&E confirmed that the U251 line shows the same features as GBM in terms of invasiveness, proliferation, and necrosis; in addition, IHC for hypoxic markers (HIF-1 $\alpha$ and CAIX) confirmed the presence of hypoxia especially in the inner portions of the tumors.
Moreover, the overlapping between HIF-1 $\alpha$ and luciferase staining on most of the histological sections provided a further demonstration of hypoxia-mediated luciferase expression.

Once the direct relationship between luciferase activity and HIF-1 $\alpha$ accumulation had been established, all subsequent experiments focused on the U251-HRE cells.

Animals intracranially injected with the U251-HRE cell line were also acquired by MRI and PET. MRI provided high-resolution images for assessing tumor localization, volume, and growth. Moreover, 30 days after cell injection, diffusion MRI, in the biggest tumors, revealed internal tumor necrosis, a structural modification due to hypoxia establishment. Future studies will further explore the development of necrotic tissues, using both diffusion and perfusion imaging. These studies will explore tumor vasculature and will be aimed at comparing the different imaging procedures for studying hypoxia in the same animal set.

As regards nuclear-based imaging, the U251-HRE injected mice were studied at two time points with different radiotracers: $\left[{ }^{18} \mathrm{~F}\right] \mathrm{FDG}$ for metabolic profile, $\left[{ }^{18} \mathrm{~F}\right] \mathrm{FLT}$ for cell proliferation, and $\left[{ }^{18} \mathrm{~F}\right] \mathrm{FAZA}$ for tissue hypoxia. Unlike what was reported in rats using U251 cells [37], $\left[{ }^{18} \mathrm{~F}\right] \mathrm{FDG}$ uptake was found to be close to normal brain tissue levels (data not shown). This difference may be due to the limited spatial resolution of YAP-(S)PET in detecting mouse brain lesions using tracers like $\left[{ }^{18} \mathrm{~F}\right] \mathrm{FDG}$ that show high background uptake in the brain. Indeed, $\left[{ }^{18} \mathrm{~F}\right] \mathrm{FLT}$ uptake, reflecting tumoral proliferation and showing a very low background signal, was clearly detectable from the early time point and increased over time, as also demonstrated by ex vivo correlation with the Ki67 marker [37, 38].

Tumor hypoxia was assessed in vivo by PET with $\left[{ }^{18} \mathrm{~F}\right] \mathrm{FAZA}$, a 2-nitroimidazole compound whose tumor-tobackground ratio is higher than those of other 2nitroimidazoles [39], and which we have previously evaluated in different tumor models [40].

In U251-HRE-injected mice, $\left[{ }^{18} \mathrm{~F}\right] \mathrm{FAZA}$ uptake within tumor lesions was detectable only in the acquisitions performed 30 days after cell injection. The results were very similar to those obtained with the FLI analysis and therefore validated this cell model for the study of hypoxia establishment and progression also by PET.

\section{Conclusions}

In conclusion, our data suggest that the U251-HRE cell line, with luciferase activity as biomarker, can be used to evaluate HIF- $1 \alpha$ activity in both in vitro and in vivo longitudinal studies geared at improving therapeutic approaches. In the near future, in fact, the U251-HRE model will be used to evaluate tumor hypoxia with multiple imaging modalities, with a view to opening up new avenues for the planning of personalized treatments [41, 42]. Translational molecular imaging modalities, indeed, offer the possibility of cross- 
validating preclinical optical imaging data with small-animal PET and MRI results, as a preliminary approach that may facilitate the transfer of the obtained results and specific imaging procedures into the clinical setting.

Acknowledgments. Lo Dico was supported by a fellowship from the Doctorate School of Molecular Medicine, University of Milan, Milan, Italy. The authors wish to thank Dr. G. Melillo for providing the engineered U251 cells, Drs. S. Todde and C. Monterisi for producing the radiopharmaceuticals. The authors are also grateful to Ms. Catherine Wrenn for her advice and skilful editorial support. This was an Eurobioimaging proof of concept study (Facility n. 45, WP8). We thank the IRCCS Cà Granda Foundation for allowing the use of the imaging system IVIS Spectrum/CT. This work was supported in part by regional AIRC Lombardia Molecular Imaging 2008 project no. 6278 and FP7 funded INSERT project (HEALTH-2012INNOVATION-1, GA305311).

Conflict of Interest. The authors declare that they have no conflict of interest.

\section{References}

1. Rankin EB, Rha J, Unger TL et al (2008) Hypoxia-inducible factor (HIF)-2 regulates vascular tumorigenesis in mice. Oncogene 27:53545358

2. Ivan M, Kaelin WG Jr (2001) The von Hippel-Lindau tumor suppressor protein. Curr Opin Genet Dev 11:27-34, Review

3. Jaakkola P, Mole DR, Tian YM et al (2001) Targeting of HIF-alpha to the von Hippel-Lindau ubiquitylation complex by O2-regulated prolyl hydroxylation. Science 292:468-472

4. Cho S, Choi YJ, Kim JM et al (2001) Binding and regulation of HIF-1 by a subunit of the proteasome complex, PSMA7. FEBS Lett 498:6266

5. Cockman ME, Masson N, Mole DR et al (2000) Hypoxia inducible factor-alpha binding and ubiquitylation by the von Hippel-Lindau tumor suppressor protein. J Biol Chem 275:25733-25741

6. Jiang BH, Rue E, Wang GL, Roe R, Semenza GL (1996) Dimerization, DNA binding, and transactivation properties of hypoxia-inducible factor 1. J Biol Chem 271:17771-17778

7. Lal A, Peters H, St Croix B (2001) Transcriptional response to hypoxia in human tumors. J Natl Cancer Inst 93:1337-1343

8. Semenza G (2002) Signal transduction to hypoxia-inducible factor 1. Biochem Pharmacol 64:993-998

9. Ivan M, Kondo K, Yang H et al (2001) HIFalpha targeted for VHLmediated destruction by proline hydroxylation: implications for $\mathrm{O} 2$ sensing. Science 292:464-468

10. Candolfi CJF, Nichols WS et al (2007) Intracranial glioblastoma models in preclinical neuro-oncology: neuropathological characterization and tumor progression. J Neurooncol 85:133-148

11. Radaelli CR, Patton V et al (2009) Immunohistopathological and neuroimaging characterization of murine orthotopic xenograft models of glioblastoma multiforme recapitulating the most salient features of human disease. Histol Histopathol 24:879-891

12. Jacobs VL, Valdes PA, Hickey WF, De Leo JA (2011) Current review of in vivo GBM rodent models: emphasis on the CNS-1 tumor model. ASN Neuro 3:e00063. doi:10.1042/AN20110014

13. Louis DN, Seizinger BR (1994) Genetic basis of neurological tumors. Baillieres Clin Neurol 3:335-352, Review

14. Van Meir EG, Hadjipanayis CG, Norden AD et al (2010) Exciting new advances in neuro-oncology: the avenue to a cure for malignant glioma. CA Cancer J Clin 60:166-193, Review

15. Koul TY, Shen R et al (2006) PTEN enhances TNF-induced apoptosis through modulation of nuclear factor-kappaB signaling pathway in human glioma cells. Biochem Biophys Res Commun 2:463-471

16. Furnari FB, Fenton T, Bachoo RM (2007) Malignant astrocytic glioma: genetics, biology, and paths to treatment. Genes Dev 21:2683-2710, Review

17. Koul D, Shen R, Bergh S (2006) Inhibition of Akt survival pathway by a small-molecule inhibitor in human glioblastoma. Mol Cancer Ther 3:637-644
18. Gheysens O, Chen IY, Rodriguez-Porcel M et al (2011) Non-invasive bioluminescence imaging of myoblast-mediated hypoxia-inducible factor-1 alpha gene transfer. Mol Imaging Biol 6:1124-1132

19. Szentirmai O, Baker CH, Lin N et al (2006) Noninvasive bioluminescence imaging of luciferase expressing intracranial U87 xenografts: correlation with magnetic resonance imaging determined tumor volume and longitudinal use in assessing tumor growth and antiangiogenic treatment effect. Neurosurgery 2:365-372

20. Rapisarda A, Zalek J, Hollingshead M et al (2004) Schedule-dependent inhibition of hypoxia-inducible factor-1alpha protein accumulation, angiogenesis, and tumor growth by topotecan in U251-HRE glioblastoma xenografts. Cancer Res 19:6845-6848

21. Rapisarda A, Hollingshead M, Uranchimeg B et al (2009) Increased antitumor activity of bevacizumab in combination with hypoxia inducible factor-1 inhibition. Mol Cancer Ther 7:1867-1877

22. Rapisarda A, Uranchimeg B, Scudiero DA et al (2002) Identification of small molecule inhibitors of hypoxia-inducible factor 1 transcriptional activation pathway. Cancer Res 15:4316-4324

23. MAES W, Deroose C, Reumers V (2009) In vivo bioluminescence imaging in an experimental mouse model for dendritic cell based immunotherapy against malignant glioma. J Neurooncol 91:127-139

24. Reischl G, Ehrlichmann W, Bieg C et al (2005) Preparation of the hypoxia imaging PET tracer $\left[{ }^{18} \mathrm{~F}\right] \mathrm{FAZA}$ : reaction parameters and automation. Appl Radiat Isot 62:897-901

25. Martin SJ, Eisenbarth JA, Wagner-Utermann U (2002) A new precursor for the radiosynthesis of $\left[{ }^{18}\right.$ F]FLT. Nucl Med Biol 29:263-273

26. Arsham AM, Plas DR, Thompson CB, Simon MC (2002) Phosphatidylinositol 3-kinase/Akt signaling is neither required for hypoxic stabilization of HIF-1 alpha nor sufficient for HIF-1-dependent target gene transcription. J Biol Chem 277:15162-15170

27. Höckel M, Vaupel P (2001) Biological consequences of tumor hypoxia. Semin Oncol 28:36-41, 2 Suppl 8

28. Yuan Y, Hilliard G, Ferguson T, Millhorn DE (2003) Cobalt inhibits the interaction between hypoxia-inducible factor- $\alpha$ and von Hippel-Lindau protein by direct binding to hypoxia-inducible factor- $\alpha$. J Biol Chem 278:15911-15916

29. Demidenko ZN, Rapisarda A, Garayoa M et al (2005) Accumulation of hypoxia-inducible factor $1 \alpha$ is limited by transcription-dependent depletion. Oncogene 30:4829-4838

30. Stiehl DP, Wirthner R, Köditz J et al (2006) Increased prolyl 4hydroxylase domain proteins compensate for decreased oxygen levels: evidence for an autoregulatory oxygen-sensing system. J Biol Chem 33:23482-23491

31. Mottet D, Dumont V, Deccache Y et al (2003) Regulation of hypoxiainducible factor-1alpha protein level during hypoxic conditions by the phosphatidylinositol 3-kinase/Akt/glycogen synthase kinase 3beta pathway in HepG2 cells. J Biol Chem 33:31277-31285

32. Görlach A (2009) Regulation of HIF-1alpha at the transcriptional level. Curr Pharm Des 33:3844-52, Review

33. Zundel W, Schindler C, Haas-Kogan D et al (2000) Loss of PTEN facilitates HIF-1-mediated gene expression. Genes Dev 14(4):391396

34. Zhong H, Chiles K, Feldser D et al (2000) Modulation of hypoxiainducible factor 1 expression by the epidermal growth factor/phosphatidylinositol 3-kinase/PTEN/AKT/FRAP pathway in human prostate cancer cells: implications for tumor angiogenesis and therapeutics. Cancer Res 6:1541-1545

35. Cantley LC, Neel BG (1999) New insights into tumor suppression: PTEN suppresses tumor formation by restraining the phosphoinositide 3-kinase/AKT pathway. Proc Natl Acad Sci USA 96:4240-4245

36. Rong Y, Hu F, Huang R et al (2006) Early growth response gene-1 regulates hypoxia-induced expression of tissue factor in glioblastoma multiforme through hypoxia-inducible factor-1-independent mechanisms. Cancer Res 66:7067-7074

37. Corroyer-Dulmont A, Peres EA, Petit E et al (2013) Detection of glioblastoma response to temozolomide combined with bevacizumab based on \{micro\}MRI and \{micro\}PET imaging reveals $\left[{ }^{18} \mathrm{~F}\right]$-fluoro-Lthymidine as an early and robust predictive marker for treatment efficacy. Neuroncology 1:41-56

38. Chen W, Cloughesy T, Kamdar N et al (2005) Imaging proliferation in brain tumors with ${ }^{18}$ F-FLT PET: comparison with 18 F-FDG. J Nucl Med 46:945-952

39. Reischl G, Dorow DS, Cullinane C et al (2007) Imaging of tumor hypoxia with [124I]IAZA in comparison with $\left[{ }^{18} \mathrm{~F}\right] \mathrm{FMISO}$ and 
$\left[{ }^{18}\right.$ F]FAZA-first small animal PET results. J Pharm Pharm Sci 10:203211

40. Valtorta S, Belloli S, Sanvito F et al (2013) Comparison of ${ }^{18} \mathrm{~F}$ fluoroazomycin-rabinofuranoside and ${ }^{64} \mathrm{Cu}$-diacetyl-bis(N4methylthiosemicarbazone) in preclinical models of cancer. J Nucl Med 54:1-7. doi:10.2967/JNUMED.112.111120
41. Randy LJ (2009) Brain tumor hypoxia: tumorigenesis, angiogenesis, imaging, pseudoprogression, and as a therapeutic target. J Neurooncol 92:317-335, Review

42. Stell A, Biserni A, Della Torre A (2007) Generation of novel animal model systems to study cancer progression and therapy. Int J Biochem Cell Biol 39(7-8):1288-1296, Review 